\title{
Neuroscience on the brain
}

The pilgrimage to the annual Society for Neuroscience meeting will convene in Los Angeles from the 7th through the 12th of November - readers may save their legs by targetting some of the items featured here.

\author{
Current Protocols in Neuroscience \\ From John Wiley \& Sons \\ An up-to-date collection of \\ neuroscience methods
}

The publication draws from techniques in neurophysiology, neuroanatomy, neuropharmacology and behavioural neuroscience to meet the needs of researchers in the full range of disciplines involved in studying the brain, nervous system, and corresponding behaviours. According to Wiley, Current Protocols in Neuroscience features carefully edited techniques with troubleshooting tips and helpful comments that come from extensive experience in using these protocols. Each feature provide step-by-step guide through methods. Moreover, quarterly updates are available in looseleaf and electronic formats and should help to keep laboratories up to date with the latest developments in this rapidly changing field. Forthcoming coverage includes gene expression in transgenic mice using neural promoters, generation and utilization of phosphorylation state-specific antibodies to investigate neuronal signaling pathways, an overview of the baculovirus expression system and the measurement of chloride movement in neuronal preparations, to name a few.

Reader Enquiry No. 100

\section{Human prion $\mathrm{Pr}_{\text {27-30 }}$ peptide $\mathrm{pAb}$ \\ From Chemicon www.chemicon.com A goat polyclonal antibody to the human, prion $\operatorname{PrP}_{27-30}$ peptide}

This antibody is made against a synthetic peptide that corresponds to amino acids 79-97 of the amino terminus of the human prion protein $\mathrm{PrP}_{27-30}$. This has been shown to be immunoreactive to the peptide by ELISA. Moreover, the antibody will immunolabel amyloid plaques in formalinfixed paraffin sections from CJD brain sections. This antibody should prove useful to scientists and neuropathologists who study the pathogenesis of prion diseases.

Reader Enquiry No. 101

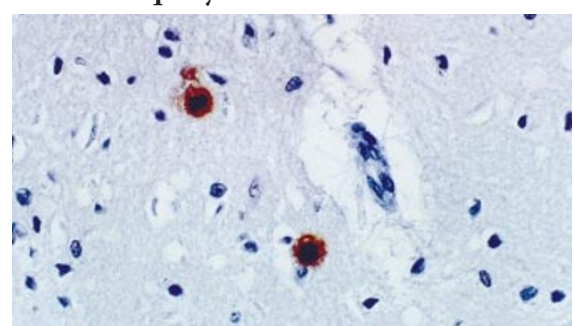

Getting your goat, polyclonal antibody, that is, to human prion peptide $\mathrm{PrP}_{27-30}$.

\section{NT-4 $E_{\max }$ immunoassay \\ From Promega www.euro.promega.com/uk \\ A system for sensitive and quantitative detection of neurotrophic factor-4 (NT-4) in an antibody sandwich format}

Soluble NT-4 is captured with an antihuman, NT-4 polyclonal antibody and then binds a second, specific anti-NT-4 monoclonal antibody. This monoclonal antibody is detected using a species-specific antibody conjugated to horseradish peroxidase. The antibodies used in this assay provide specific detection of NT-4 to a minimum of $9.4 \mathrm{pg}$ $\mathrm{ml}^{-1}$ and demonstrate less than three per cent crossreactivity with other related neurotrophic factors. The immunoassay system can be used to quantitate NT-4 in tissue culture supernatants, plasma, serum and tissue extracts. Available in two sizes, with two or five 96-well ELISA plates, this system can be configured as desired. Other immunoassays in this range include TGF $\beta 1$ and TGF $\beta 2$, BDNF, GDNF, gp130, NGF and NT-3.

Reader Enquiry No. 102

\section{Reagents for Alzheimer's research \\ From Serotec}

www.serotec.co.uk

Human polyclonal antibodies and

recombinant proteins for researching

Alzheimer's disease

Products currently available from the company for this area of research include presenilin-1, amyloid- $\beta$, amyloid- $\beta$ precursor and apolipoprotein E. Many of these products are suitable for use in a wide range of applications, including immunocytochemistry, ELISA, immunoprecipitation and western blotting.

\section{Reader Enquiry No. 103}

\section{Synuclein antibodies}

From Affiniti Research Products

\section{www.affiniti-res.com}

Inclusions known as Lewy bodies may

contribute significantly to neuronal malfunction and finally to cell death

Found in Parkinson's disease, Lewy bodies are known to contain accumulations of $\alpha$-synuclein, ubiquitin, and various proteinaceous elements of the ubiquitinproteasome pathway. Polyclonal antibodies specific to $\alpha$ - and $\beta$-synuclein have recently been developed and added to the company's listing of products for brain disease research. The peptide-derived antibodies are suitable for use in western blotting and immunohistochemical applications. In

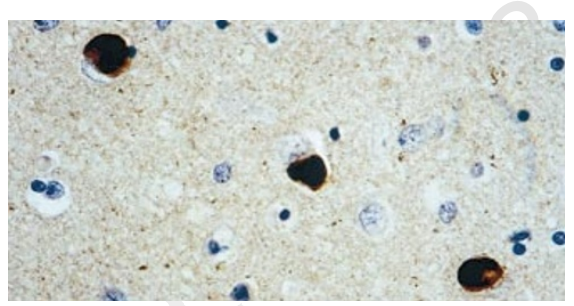

New synuclein antibodies offered by Affiniti.

addition to the synuclein antibodies, Affiniti offers some 40 antibodies to proteasome subunits and ubiquitin-related compounds, together with a comprehensive range of substrates and proteasome inhibitors. Also offered are antibodies to neurofilaments, tau, tau-protein kinase (GSK3 $\beta$ ), and other antigens that are of importance to understanding the mechanisms of neurodegeneration.

Reader Enquiry No. 104

\section{Mdr1a/b double knockout mouse}

From Taconic

www.taconic.com

A new double knockout mouse is offered for research into the central nervous system

This mouse contains a disruption of multidrug resistance genes mdrla and $m d r l b$, it has a functional deficiency in the blood brain barrier and is applicable to a wide range of central nervous system research. It should prove useful for studies involving neurotoxicology, drug transport, chemotherapy, oral bioavailability and multi-drug resistance. The mdrla/b double knockout mouse was generated by sequential gene targetting of embryonic stem (ES) cells in the laboratory of Alfred Schinkel et al. at the Netherlands Cancer Institute. According to Taconic, these mice exhibit normal development, viability and fertility. Moreover, the mdrla/b double knockout model has shown an increase in the accumulation of digoxin in the brain and testis (male) - this is similar to the mdrla knockout mouse model. The mdrla/b mouse also showed a significant increase in accumulation of digoxin in the ovaries and adrenal glands, over plasma levels and over

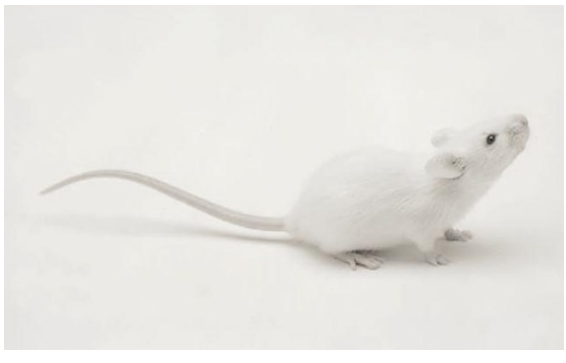

Double trouble? Not with this double knockout. 
levels in wild type mice, states Taconic. The mdrla/b knockout mouse is available on a FVB background.

Reader Enquiry No. 105

\section{Imaging systems}

\section{AlphaEase 4.0 software}

From Alpha Innotech www.alphainnotech.com Imaging software for the AlphaImager and Chemilmager systems

Centred on 32-bit architecture, this program supports long file names, enhanced dialogue boxes, and a more familiar look and feel to the software features. Features include MovieMode, camera exposure control from $0.033 \mathrm{~s}$ to $4 \mathrm{~h}$, nine levels of automatic image enhancement, zoom/pan controls, histograms, filters and false colours. Analysis tools include one-dimensional densitometry, molecular weights, high-density array analysis, one-dimensional multi-lane analysis, two-dimensional spot densitometry analysis, manual and automatic count for colonies and cells, gel scoring and ruler functions. This new architecture yields more robust compatibility with most Windows applications, as well as enhanced multitasking. AlphaEase 4.0 also boasts new updates that

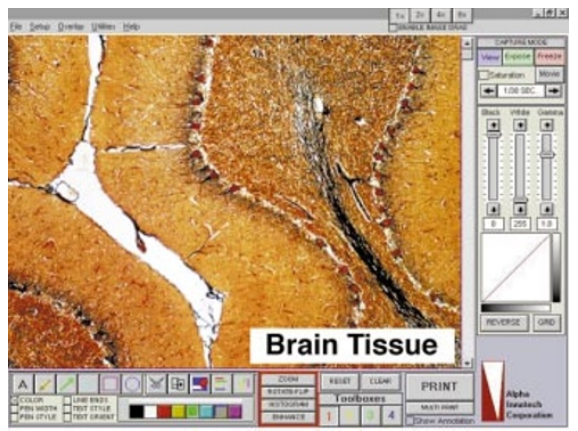

AlphaEase imaging software from AlphaInnotech.

include 8- and 16-bit grayscale file import, 24-bit colour, higher resolution file import and TWAIN acquisition.

Reader Enquiry No. 106

\section{New technical notes}

From Bio-Rad www.bio-rad.com

Two new notes that cover multi-photon imaging of biological tissue

Technical note five summarizes the benefits of non-descanned (external) detectors in collecting scattered light and improving sensitivity, allowing deeper optical sectioning and detection of faint fluorescent signals.

\section{ADVERTISEMENT}



Western Blotting Applications Sopersinnal West Pics Chemiluminescenl Substrate* Pcogram laved sensitivty at an anazingly economical pr c3 Prosust $+340 \mathrm{so日}, 560 \mathrm{ml}$

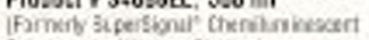
Sedistus for Wostim Eastingi Supersinnal West Dura Extended Duraticn Substrate* 24-hour signa duration and super sersithity

Prossut : 34075 $160 \mathrm{mI}$

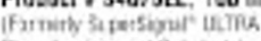



Sapersignal" West Femlo Meximem Seasifvity Substrate* The most intense scral ard the greatest sensitivity altsinable sith a comiluminestent substrate Prosat +36085 . $100=1$

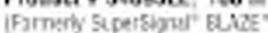

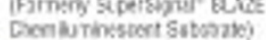

H Super Spectrum of Possibilities

Pierce SuperSignal Chemiluminescent Substrates for all your protein research needs.

ELISA

SuperSignal" ELISA Pieo Chemiluminessent Substrate* Piccoram tevel detection for plate-based assiys

Frndact o 37jiet. $100 \mathrm{r}$

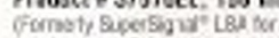

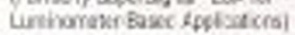
SuperSigral" ELISA Fanto Masinum Sensitivity Substraie* A more senstive substrate with enhanced low-and inearit; Fraduct 2 37JTSEL, 100

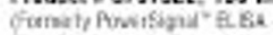
Cour isrinseicut Sitprale "Pawi henerio

Call ter your FREE Substraxs Selecjon Guife Paster! Call 300-874-3723 far croduct istormation or 815.963 .0747 190 the nente of your local distibitar
It also illustrates the design of the external detector on the MRC-1024MP system and lists the full range of available filters. On the same subject, technical note number seven describes the advantages of using femtosecond, pulsed lasers for achieving deeper imaging into tissue with flexibility in the use of available power. Multi-photon imaging is increasingly used for studying biological tissue as it allows deeper imaging into samples than other methods and enables the study of living samples for longer periods of time than previously possible, says Bio-Rad. Reader Enquiry No. 107

\section{PhoTurbo}

From Life Science Resources www.lsr.co.uk Fluorescence detection for simultaneous realtime photometry and electrophysiology

According to the manufacturer, PhoTurbo provides the very high temporal resolution in data acquisition for experiments that the latest optical probes in high speed neurobiological research applications. It has two-in-one functionality, as the system is configured with both PhoCalTurbo and PhoClampTurbo on a single platform. These systems enable both chart-recorder-like data acquisition and triggered sweep acquisition, with full experimental control and on-line viewing of data. PhoTurbo has 16-bit precision and dynamic range to acquire both fluorescence and analog data at rates up to 64 $\mathrm{kHz}$. According to the manufacturer, highsensitivity photon-detection measurement makes it possible to record and display online even the fastest of physiological events in real time. The system measures optical probes used in the detection of membrane potential or ions, such as calcium sodium and magnesium, which can be combined with such analog patch clamp measurements as voltage and current, or with extracellular $\mathrm{pH}, \mathrm{O}_{2}$ tension and temperature. PhoTurbo is a complete, integrated system, operating under Windows 95 with full 32-bit functionality. It includes photomultiplier tubes, all associated electronics, acquisition hardware, power supplies and workstation.

Reader Enquiry No. 108

\section{DP10 digital camera}

From Olympus www.olympus-europa.com A digital photomicrography system for high performance at a more reasonable price

The DP10 digital, colour camera has a progressive $1.7-\mathrm{cm}$ CCD sensor with over 1.4 million pixels $(1,280 \times 1,024$ pixels $)$. However, image resolution is not artificially increased or interpolated by software as is common with other cameras within this price range. Instead, every pixel of the CCD sensor is represented directly in the digital picture. The technology is said to ensure that pictures are sharp with natural colour 